\title{
DIFERENCIAÇÃO ESPECTRAL DE SOLOS UTILIZANDO DADOS OBTIDOS EM LABORATÓRIO E POR SENSOR ORBITAL ( ${ }^{1}$ )
}

\author{
PETERSON RICARDO FIORIO $\left(2^{*}\right)$; JOSÉ A. M. DEMATTÊ $\left({ }^{3}\right)$; \\ MARCOS RAFAEL NANNI $\left({ }^{4}\right)$; ANTONIO ROBERTO FORMAGGIO $\left({ }^{5}\right)$
}

\begin{abstract}
RESUMO
Um grande número de amostras de solos seria necessário para delimitar com precisão os limites das unidades de solos. Assim, técnicas que possam auxiliar em levantamentos pedológicos como o sensoriamento remoto tem provado ser de grande valia. O objetivo foi avaliar a possibilidade de diferenciar solos por meio de dados espectrais em laboratório e orbitais. Para o trabalho foi escolhida uma área de 473 ha com solo exposto, proveniente de dois materiais de origem distintos, o arenito com cimento argiloso e o basalto. Foi estabelecida uma grade $(100 \mathrm{~m} \times 100 \mathrm{~m})$ em toda a área de estudo, totalizando 473 pontos de amostragem, todos georreferenciados. Para cada ponto da grade, foram coletadas amostras de solo nas profundidades 0-20 cm (superfície, A) e 80-100 cm (subsuperfície, B). A partir dos resultados das análises químicas e físicas e descrição de perfis, gerou-se o mapa de solos, e posteriormente, cada unidade desse mapeamento foi diferenciada por textura. As reflectâncias de laboratório foram obtidas com o espectroradiômetro IRIS, na faixa de 450 a $2500 \mathrm{~nm}$. As reflectâncias orbitais foram obtidas após o processamento de uma imagem TMLandsat-5, para os pontos de amostragem. Equações discriminantes foram geradas partindo-se dos dados espectrais por tratamento estatístico, que selecionou entre as setenta variáveis espectrais em laboratório, cinquenta e seis, sendo selecionadas para o modelo espectral orbital as seis bandas reflectivas do sensor TM. As equações geradas foram testadas, obtendo-se matrizes de confusão, onde ocorreu um acerto de $81 \%$ para os dados espectrais em laboratório e $40 \%$ de concordância para os dados orbitais.
\end{abstract}

Palavras-chave: Equações discriminantes, reflectância, sensoriamento remoto, mapeamento de solos.

( $\left.{ }^{1}\right)$ Recebido para publicação em 9 de abril de 2009 e aceito em 3 de novembro 2009. Extraído da Tese de Doutorado apresentada pelo primeiro autor à Escola Superior de Agricultura “Luiz de Queiroz" - ESALQ/USP, Piracicaba (SP).

$\left(^{2}\right)$ Departamento Engenharia Rural, ESALQ/USP Caixa Postal 09, 13418-900 Piracicaba (SP). E-mail: fiorio@esalq.usp.br; $\left.{ }^{*}\right)$ Autor correspondente.

$\left({ }^{3}\right)$ Departamento de Solos e Nutrição de Plantas, ESALQ/USP, Piracicaba (SP). E-mail: jamdemat@esalq.usp.br;

$\left({ }^{4}\right)$ Departamento de Agronomia, Universidade Estadual de Maringá - UEM,Caixa Postal 331, 87020-900 Maringá (PR). E-mail: mrnanni@uem.br;

$\left(^{5}\right)$ Instituto Nacional de Pesquisas Espaciais (INPE/MCT) Caixa Postal 515, 12201-970 São José dos Campos (SP). E-mail: formag@dsr.inpe.br. 


\title{
ABSTRACT \\ SPECTRAL DIFFERENTIATION AMONG SOILS USING SPECTRAL DATA FROM LABORATORY AND ORBITAL SENSOR
}

\begin{abstract}
A huge amount of soil samples would be necessary to determine with precision the limits of soil units. Techniques that would help on soil surveys such as the remote sensing ones have been proved to be of great value. The objective of this work was to evaluate the possibility of differentiating soils using spectral data from laboratory and satellite sensors. A study area with soils originated from two different parent materials, sandstone with loamy cement and basalt, was selected. A grid (100 m x $100 \mathrm{~m})$ was used, totalizing 473 sampling points in the depths $0-20 \mathrm{~cm}$ (superficial, A) and 80-100 cm (subsuperficial, B). A soil map of the area with 28 mapping units was generated. The laboratory spectra (450 to $2500 \mathrm{~nm})$ were obtained with an IRIS spectroradiometer. The orbital reflectance was obtained by image processing and overlaying of the TM-Landsat- 5 bands in the sampling points. Discriminant equations were generated by statistical treatment with the SAS software. From the seventy initial variables from laboratory data, fifty six were selected to generate the models of the equations; for orbital data, six of the seven TM bands were selected. The equations were tested and the confusion matrix were obtained. Discriminant equations from laboratory data presented $81 \%$ success and the equations with orbital data $40 \%$, however, for the satellite data it was verified a good efficiency for separating soils from different parent materials.
\end{abstract}

Key words: Discriminant equations, reflectance, remote sensing, soil mapping.

\section{INTRODUÇÃO}

O conhecimento dos atributos dos solos, bem como sua distribuição espacial e mapeamento, constitui-se em ferramenta imprescindível para o adequado manejo ambiental. Essas informações, quando manejadas de forma correta, tornam- se uma ferramenta importante para se prolongar e até mesmo aumentar a capacidade produtiva das áreas agrícolas. Porém, Fiorio et al. (2000) verificaram que o avanço desordenado da ocupação das terras com cana-deaçúcar, sem um planejamento prévio, em solos com alta suscetibilidade à erosão, causou assoreamento em uma represa no município de Piracicaba (SP). Segundo NANNI et al. (2004), discussões sobre a necessidade de agregação de esforços e trabalhos em conjunto que possam contribuir para a avaliação quantitativa dos solos agrícolas tem merecido destaque em artigos e publicações específicas. Alguns desses artigos propõem melhor compreensão do comportamento espectral dos solos por meio de sensores remotos. StONER e BAUMgardenER, (1981) e Formaggio et al. (1996) demonstraram a possibilidade da caracterização dos solos por sensores remotos no nível terrestre (laboratório e campo). Coleman et al. (1993) consideram que a avaliação do comportamento espectral dos solos poderia ser até mesmo por imagens de satélite, auxiliar mais rapidamente na obtenção de informações relativas à caracterização e ao mapeamento de solos. Com o advento de novos sensores e a intensificação da utilização da tecnologia na agricultura, o desenvolvimento de técnicas de sensoriamento remoto aplicadas ao estudo de solos também se torna função importante, como descreve Demattê (2001) sobre a utilização de novas tecnologias no auxílio dos mapeamentos de solos.
BAPTISTA et al. (1999), usando metodologia baseada na manipulação matemática de bandas do sensor hiperespectral AVIRIS, geraram mapa de teores de argila, que pode auxiliar nos mapeamentos de solos. A fundamentação dos estudos nessa área se baseia na individualização espectral de cada solo, ou seja, cada solo representa uma assinatura espectral, decorrente de seus atributos, com informações em determinado comprimento de onda ou em todo espectro eletromagnético. Demattê e Garcia (1999b) observaram que solos com teores semelhantes de ferro total possuíam diferenças significativas entre os comprimentos de onda de 400 a $800 \mathrm{~nm}$ e 800 a 1100 $\mathrm{nm}$. Solos de textura mais arenosa tiveram maior reflectância em todo o espectro eletromagnético estudado (450 a $2500 \mathrm{~nm}$ ), que solos de textura mais argilosa, com menor reflectância (Demattê e NANNI, 2003). Odeh e Macbratney (2000) comprovaram que a utilização do sensoriamento remoto e técnicas estatísticas foram as mais eficientes e menos onerosas, na diferenciação entre unidades de mapeamento. A discriminação de unidades de solos baseia-se no pressuposto de que cada classe possui características que a difere das demais e, portanto, é taxonomicamente individualizada; como a resposta espectral dos solos também é uma característica individualizadora, supõe-se que o uso desta variável possa separar as classes de solos, auxiliando nos levantamentos pedológicos (NANNI, et al., 2004). A análise discriminante é como um método auxiliar nos levantamentos dos solos (Webster e Oliver, 1990). Webster e Burrough (1974), Demattê e Garcia (1999a), Demattê e Nanni (2003) e Fiorio e Demattê (2009) têm demonstrado a possibilidade de predizer classes de solos e sua separabilidade na paisagem por meio da análise multivariada. Levantamentos pedológicos e 
de atributos dos solos têm sido realizados com a otimização da análise discriminante (SINOWSKI e Auerswald, 1999; KravchenKo et al., 2002). Demattê e GARCIA (1999b) concluíram que as análises discriminantes foram 100\% eficientes na separação do Latossolo Bruno e do Nitossolo Vermelho, utilizando dados espectrais em laboratório, com 13 bandas, entre $684 \mathrm{~nm}$ e $2.500 \mathrm{~nm}$. NANNI et al. (2004), utilizando dados espectrais em laboratório, trabalhando com 43 bandas espectrais, em uma área com 18 unidades de solos, verificaram pelas análises discriminantes que um acerto superior a $80 \%$ pode ser obtido na diferenciação espectral de solos. Para a mesma área, porém com dados espectrais orbitais, NANNI (2000) obteve acerto de $40 \%$, e quando os solos foram agrupados em função do material de origem, o acerto para os dados orbitais passou para $70 \%$. Demattê et al. (2000) concluíram que as análises discriminantes obtidas com radiometria de laboratório discriminaram $100 \%$ das 26 unidades estudadas e $99,3 \%$ para as discriminantes obtidas com dados orbitais do sensor TM-Landsat 5. KRAVCHENKO et al. (2002) revelam como desvantagem da análise discriminante no mapeamento das propriedades dos solos é que a localização geoespacial não pode ser utilizada no desenvolvimento de modelos preditores. A distribuição das propriedades dos solos na paisagem, segundo SinOwSKI e AURERSwald (1999), é controlada pelo relevo, material parental, clima, organismos e tempo. Tem-se como hipótese do trabalho que os dados espectrais podem auxiliar em levantamentos de solos otimizando o tempo e custos, devido à rapidez na coleta dos dados, e grande número de amostras que podem ser processadas. O objetivo do trabalho foi o de desenvolver e avaliar uma metodologia para discriminação das classes de solos a partir de suas respostas espectrais, utilizando-se sensores em laboratório e orbital.

\section{MATERIAL E MÉTODOS}

\section{Caracterização da área de estudo}

A área localiza-se ao sudoeste do Estado de São Paulo, na região de Barra Bonita. É delimitada pelas coordenadas geográficas $22^{\circ} 26^{\prime} 02^{\prime \prime}-22^{\circ} 23^{\prime} 16^{\prime \prime}$ Latitude Sul e $48^{\circ} 31^{\prime} 24^{\prime \prime}$ - $48^{\circ} 27^{\prime} 51^{\prime \prime}$ Longitude Oeste (Figura 1). Compõe de 473 ha cultivados com canade-açúcar em altitudes variando entre 520 e 710 metros, sendo que a área possui as seguintes classes de declividade de acordo com a EMBRAPA (1999): 0-3\% com 47,59 ha, $3 \%-8 \%$ com 183,66 ha, $8 \%-20 \%$ com 237,20 ha e $20 \%-40 \%$ com 2,64 ha. O clima da região, com base na classificação de Köppen, é do tipo Cwa, clima mesotérmico com inverno seco e verão quente (Sentelhas et al., 1998). A geologia é representada pela ocorrência da Formação Serra Geral, a qual se caracteriza por compreender um conjunto de derrames de basalto, entre os quais se intercalam arenitos com as mesmas características da Formação Botucatu. Aparece ainda a Formação Itaqueri do grupo Bauru que se caracteriza por arenitos com cimento argiloso, folhelhos e conglomerados, predominando os arenitos (IPT, 1981).

\section{Mapeamento de solos}

Para a amostragem, foi estabelecida uma grade regular de $100 \mathrm{~m} \times 100 \mathrm{~m}$ e os locais dos pontos foram estaqueados, numerados, georreferenciados e coletadas amostras de solos nas profundidades de $0-20 \mathrm{~cm}$ superfície e de $80-100 \mathrm{~cm}$ subsuperfície, totalizando 946 amostras de solos (Figura 1). A cor do solo foi obtida na forma úmida com o equipamento Minolta CR 300 com chip para cor de Munsel. Foram realizadas análises químicas de acordo com a metodologia preconizada por (RAIJ e Quaggio, 1987), granulométricas e de ataque sulfúrico conforme (CAMARGo et al., 1987). Com o resultado das análises químicas, físicas e mapa planialtimétrico, foram traçadas unidades de mapeamento, sendo determinado o local para abertura de trincheiras. Dessa forma, foram descritos 11 perfis de solos, seguindo a metodologia de (Lemos e Santos, 1996). Como base para a classificação (EMBRAPA, 1999), foram obtidas as onze classes de solos: Latossolo Vermelho Distrófico (LVd), Latossolo Vermelho Eutroférrico (LVef), Latossolo Vermelho-Amarelo Distrófico (LVAd), Argissolo Amarelo Distrófico (PAd), Argissolo Vermelho-Amarelo Distrófico (PVAd), Argissolo Vermelho Distrófico (PVd), Nitossolo Vermelho Eutrófico (NVe), Nitossolo Vermelho Eutroférrico (NVef), Cambissolo Háplico Tb Eutrófico (CXbe), Cambissolo Háplico Eutroférrico (CXef) e Neossolos Quartzarênicos Órticos (RQo). Para testar a metodologia proposta e tentar verificar a sensibilidade dos dados espectrais com as características físicas, os solos foram diferenciados de acordo com as seguintes classes de textura: Arenosa (< $150 \mathrm{~g} \mathrm{~kg}^{-1}$ de argila), Média 1 (1) $(>150$ e $<250 \mathrm{~g} \mathrm{~kg}^{-1}$ de argila), Média 2 (2) $(>250 \mathrm{e}<350 \mathrm{~g}$ $\mathrm{kg}^{-1}$ de argila), Argilosa (3) (>350 e $<600 \mathrm{~g} \mathrm{~kg}^{-1} \mathrm{de}$ argila) e Muito Argilosa (4) (> $600 \mathrm{~g} \mathrm{~kg}^{-1}$ de argila), números $(1,2,3$ e 4$)$ na legenda e no texto representam as classes de textura anteriormente descritas, obtendo-se o que denominamos de "mapa detalhado por textura" da área de estudo, com 28 unidades de mapeamento (Figura 2). 


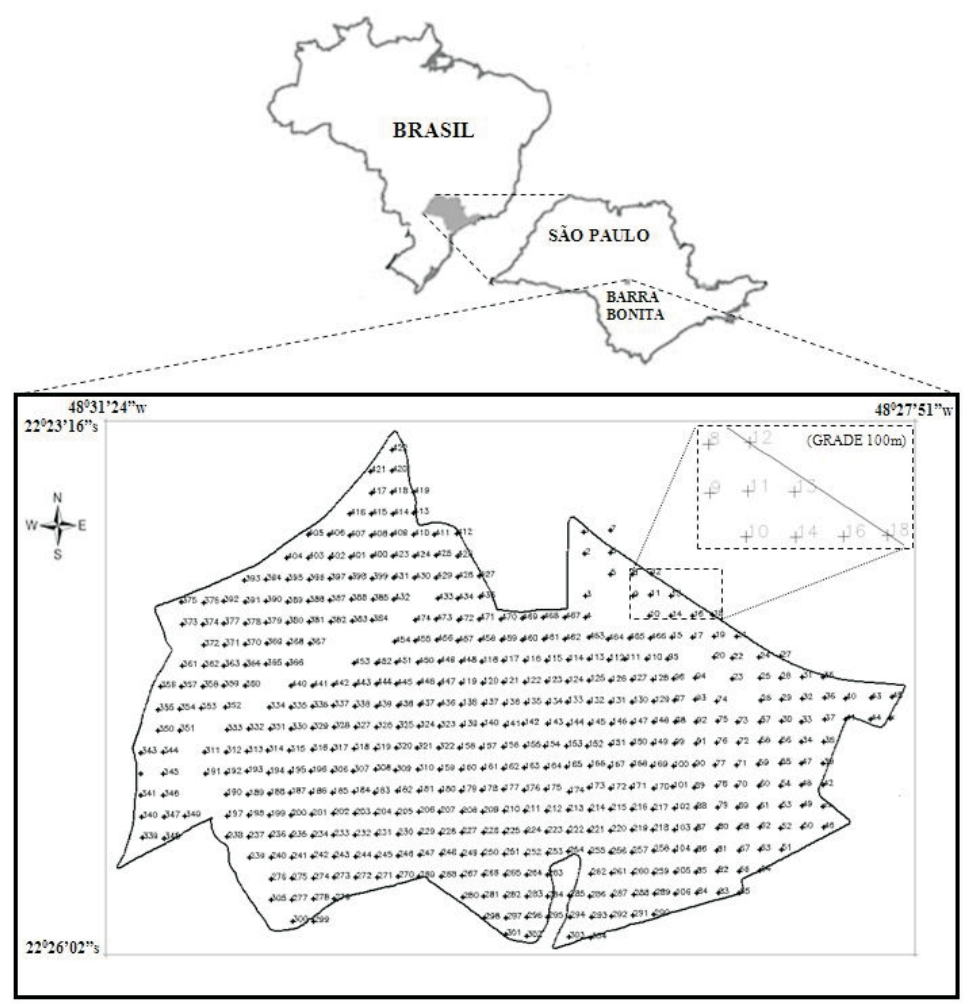

Figura 1. Localização da área de estudo e representação da grade de amostragem.

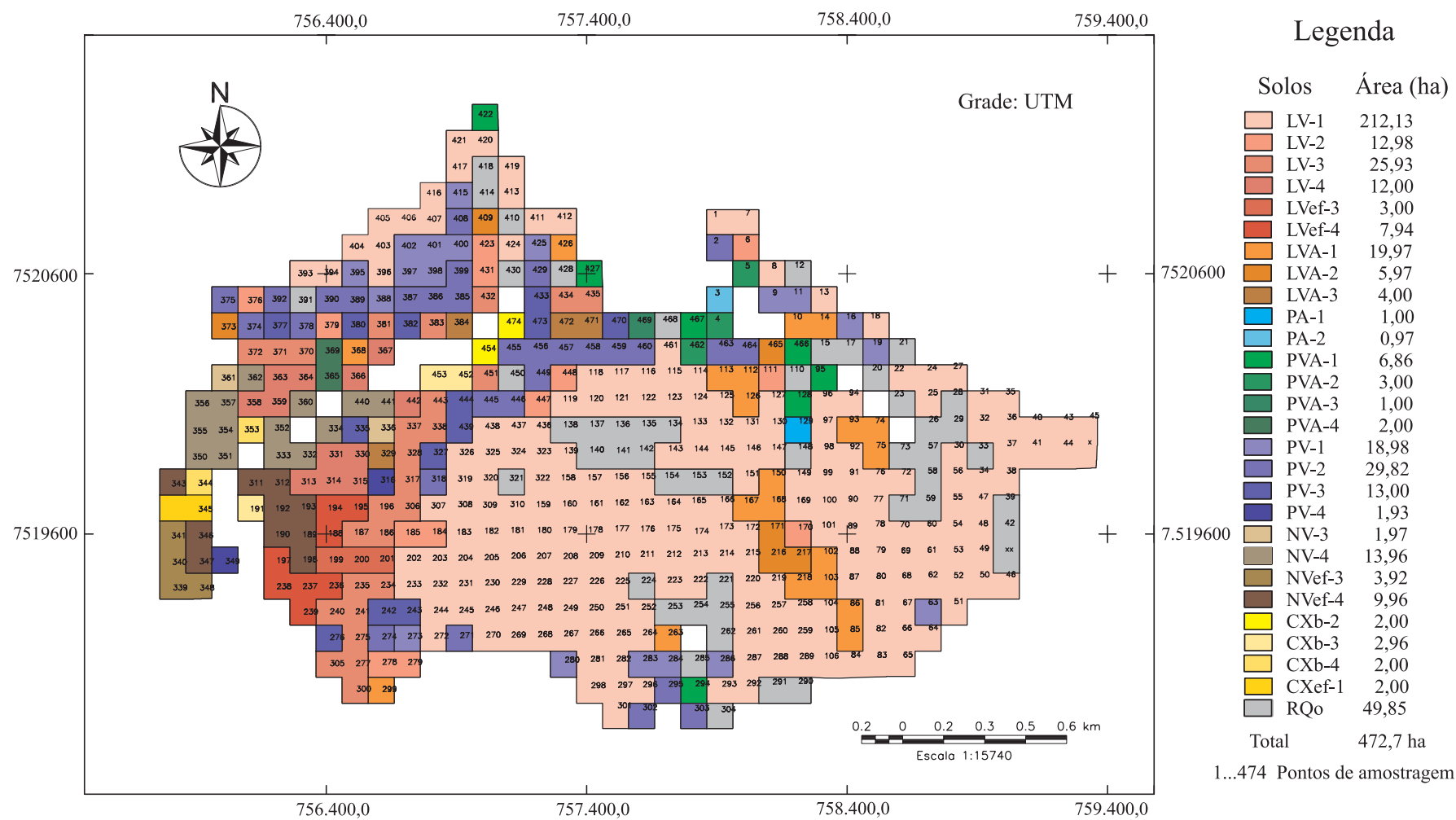

Figura 2. Representação do mapa de solos detalhado por textura. Onde (LV) Latossolos Vermelhos; (LVef) Latossolos Vermelhos Eutroférricos; (LVA) Latossolos Vermelho-Amarelos; (PA) Argissolos Amarelos ; (PVA) Argissolos Vermelho-Amarelos; (PV) Argissolos Vermelhos; (NVe) Nitossolos Vermelhos Eutróficos; (NVef) Nitossolos Vermelhos Eutroférricos; (CXb) Cambissolos Háplicos Tb; (CXef) Cambissolos Háplicos Eutroférricos; (RQo) Neossolos Quartzarênicos Órticos. Os números à frente das siglas dos solos representam texturas: 1 (média 150-250 $\mathrm{g} \mathrm{kg}^{-1}$ ); 2 (média 250-350 $\mathrm{g} \mathrm{kg}^{-1}$ ); 3 (argilosa 350-600 $\mathrm{g} \mathrm{kg}^{-1}$ ) e 4 (muito argilosa $>600 \mathrm{~g} \mathrm{~kg}^{-1}$ ). 


\section{Obtenção dos dados espectrais}

Foram utilizadas as seis bandas refletivas de uma cena TM-Landsat-5 da orbita/ponto 220/76 e o software Spring (INPE, 1999) para os processamentos de imagem. Após definida a área, foi realizado o corte na imagem no programa IMPIMA (INPE, 1999). Segundo BEN-Dor (2002), os valores de níveis de cinza obtidos das cenas do sensor TM-Landsat 5 devem ser devidamente ajustados aos efeitos atmosféricos, e posteriormente os números digitais convertidos em valores de reflectância real, ainda segundo EPIPHANIO e FormagGio (1988), a análise quantitativa de imagens digitais sobre o comportamento espectral de alvos deve ser feita com os dados em reflectância, por ser uma grandeza física intrínseca dos alvos, ao passo que os números digitais são valores transformados para a obtenção das imagens orbitais. Para a correção atmosférica, foi utilizado o modelo 5S (Simulação do Sinal do Satélite dentro do Espectro Solar), conforme proposto por TANRÉ et al. (1992) e detalhadamente descrito por Zullo Jr. (1994).

Para adequar o posicionamento da imagem com a verdade de campo, faz-se necessária a correção geométrica. Dessa forma, foram utilizadas as cartas planialtimétricas, escala 1:10.000, da Coordenadoria de Ação Regional, Divisão Cartográfica, da Secretaria de Economia e Planejamento do Estado de São Paulo. Além disso, pontos de controle obtidos no campo com equipamento GPS (Global Positioning System) com erro submétrico foram utilizados. Objetivando manter o valor do pixel o mais semelhante possível ao seu valor original, utilizou-se o método de interpolação denominado vizinho mais próximo, corrigindo apenas as distorções de escala, deslocamento ou rotação existentes entre a imagem e a projeção terrestre. Uma vez registrada, a cena foi visualizada em composição colorida R/G/B, bandas $1 / 2 / 3$ e $4 / 5 / 7$. Sobre os planos de informação "imagens", foram sobrepostos os pontos de amostragem da grade. Em cada ponto de amostragem, foram determinados os dados espectrais nas bandas TM da imagem utilizada.

Para a obtenção dos dados espectrais em laboratório, foi utilizado o sensor Infra-Red Intelligent Spectroradiometer, IRIS (GEOPHYSICAL e ENVIRONMENTAL Research Corporation, 1996), que recobre a faixa espectral entre 450 e $2.500 \mathrm{~nm}$. Todas as amostras dos solos foram secas em estufa a $45{ }^{\circ} \mathrm{C}$ por 24 horas, moídas e peneiradas (malha de $2 \mathrm{~mm}$ ), para homogeneização dos efeitos da umidade e rugosidade (EPIPHANIO et al., 1992). Posteriormente, as amostras foram acondicionadas em placas de petri e iluminadas por uma lâmpada halógena de $650 \mathrm{~W}$. Utilizou-se a placa padrão branca, com $100 \%$ de reflectância calibrada de acordo com LABSPHERE (1996). A geometria utilizada foi: $61 \mathrm{~cm}$ lâmpada-alvo e alvo-sensor: 27 $\mathrm{cm}$; inclinação da lâmpada $20^{\circ}$ ao nadir conforme padronizada por (BAUMGARDNER et al., 1985). Posteriormente, os dados de radiância foram referenciados com os dados da placa-padrão, tendose realizado uma filtragem para eliminação do excesso de ruídos e para facilitar a interpretação das curvas espectrais. A relação entre a energia refletida pelo alvo e a energia refletida pela placa de referência gerou o fator de reflectância bidirecional como sugerido por (Nicodemus et al., 1977). Foram realizadas três leituras em cada amostra, sendo utilizada a curva espectral média.

\section{Análise estatística dos dados espectrais e geração dos modelos}

Para os dados orbitais foram obtidos os dados de reflectância superficial dos solos, referentes às amostras de $0-20 \mathrm{~cm}$, das bandas do TM-Landsat5, sendo (em nm): B1: 450-520, B2: 520-600, B3: 630-690, B4: 760-900, B5: 1550-1750, B7: 2080-2350. Os dados espectrais obtidos com o IRIS em laboratório permitem maior resolução radiométrica, podendo-se estabelecer um maior número de bandas. Foram utilizadas as amostras superficiais e subsuperficiais. Foram selecionadas inicialmente 22 bandas (B) e 13 alturas (H) de bandas de absorção, seguindo metodologia de Nanni e Demattê (2001b), sendo elas (em nm): B1: 450481, B2: 481, B3: 481-596, B4: 596-710, B5: 710-814, B6: 814-975, B7: 975-1350, B8: 1350-1417, B9: 1417, B10: 1417-1449, B11: 1449-1793, B12: 1793-1831, B13: $1865-$ 1927, B14: 1927, B15: 1927-2102, B16: 2101-2139, B17: 2139-2206, B18: 2206, B19: 2206-2258, B20: 2258, B21: 2258-2389, B22: 2389-2498, H1: 469-532, H2: 532-768, H3: 768-876, H4: 876-1353, H5: 1353-1411, H6: 14111439, H7: 1439-1783, H8: 1860-1923, H9: 1923-2120, H10: 2120-2206, H11: 2206-2258, H12: 2258-2389, H13: 2389-2498. Na análise estatística dos dados foi utilizado o programa SAS (SAS, 1999). A matriz de dados foi composta para os dados de laboratório por 22 bandas e 13 diferenças de altura, para as duas profundidades, com um total de 70 variáveis. Para os dados orbitais, pelas seis bandas do sensor TMLandsat-5. A seleção das variáveis preditoras pelo sistema SAS é o mesmo para os dados orbitais e de laboratório. Em primeiro momento, foram realizadas as seleções das variáveis preditoras, em que o programa utilizado verifica, para o conjunto dos solos a serem discriminados, quais variáveis espectrais são significativas a $1 \%$ de probabilidade. Visando evitar qualquer tendenciosidade na análise, executou-se avaliação de colinearidade entre as variáveis para que não houvesse duas ou mais variáveis que pudessem estar sobrepondo-se, portanto, interferindo no processo discriminatório das classes de solos analisadas. 
Na diferenciação e caracterização dos solos, foi realizada a análise discriminante, com o objetivo de desenvolver e validar o método para a determinação das classes de solos a partir de seus dados espectrais. Neste caso, a classe de solo avaliada torna-se função de suas respostas espectrais, ou seja, classe de solo $=\int$ (Bandas espectrais e alturas), para os dados de laboratório e classe de solo $=\int$ (Bandas sensor TM-Landsat-5) para os dados orbitais. Nesse processo foi utilizado o procedimento DISCRIM, o qual segundo AfIFI et al., (2004) possibilita por meio do sistema computacional SAS a obtenção dos coeficientes das equações discriminantes. Para reforçar e validar a análise discriminante, o programa selecionou $80 \%$ das amostras para gerar o modelo, deixando $20 \%$ para testá-lo por cinquenta vezes consecutivas, gerando erros e acertos.

\section{RESULTADOS E DISCUSSÃO}

\section{Análise discriminante}

Para os dados espectrais em laboratório, das 28 classes de solos iniciais (Figura 2), foram analisadas vinte e três classes, e para as classes de solos LVef 3, LVef 4, NV 3, NV 4, CXb 2, CXb 3 e CXb 4 não foi adotada a diferenciação por textura, sendo reagrupados como LVef, $\mathrm{NV}$ e $\mathrm{CXb}$, aumentando assim a representatividade de algumas classes. Segundo NANNI (2000), é de se esperar que as classes que contenham um número muito pequeno de indivíduos tenham menor amplitude ou variabilidade de atributos. Para os dados de laboratório, dentre as setenta variáveis estabelecidas (22 bandas e 13 alturas, para as duas profundidades de amostragem), cinqüenta e seis variáveis foram selecionadas para os modelos pelo STEPEDISC do SAS (SAS, 1999) (Tabela 1). NANNI et al. (2004) obteve para os modelos 39 variáveis, das setenta variáveis estabelecidas para as mesmas profundidades de amostragem, em uma área com grande heterogeneidade de solos, diferindo assim dos resultados constatados deste trabalho, onde foi selecionado um número bem maior de variáveis em uma área com maior homogeneidade de solos (Figura 2).

O número de variáveis preditoras, bandas e alturas, selecionadas na superfície $(0-20 \mathrm{~cm})(26)$, foi menor que o número de variáveis para a subsuperfície (80-100 $\mathrm{cm})$ (30) (Tabela 1). Tal fato pode estar associado à menor diferenciação dos solos na superfície na maior parte da área de estudo, onde predominam as texturas arenosas e médias, que condizem com o teste de colinearidade, onde se espera que em áreas mais homogenias, mais de uma variável esteja se sobrepondo (Tabela 2).

Para as amostras de superfície, a banda 8 e as alturas 4 e 11 foram retiradas do modelo, pois não foram significativas a $1 \%$ de probabilidade (STEPEDISC); para a subsuperfície apenas a banda 16 foi retirada. As demais bandas e alturas que foram retiradas do modelo tiveram colinearidade, em função de que duas variáveis ou mais explicam o mesmo fator no modelo concordando com observações de BEN-DoR (2002). Para a superfície, as bandas 2, 14, 16, 18 e 20 e a altura 13 foram retiradas por colinearidade; para a subsuperfície, foram retiradas as bandas 2, 6, 9, e 18, também por colinearidade. É interessante notar que as bandas $2(481 \mathrm{~nm})$ e $18(2.206 \mathrm{~nm})$ não entraram em nenhum dos modelos, tanto para a superfície, como para a subsuperfície, o que pode ser devido a um recobrimento da banda 2 pelas bandas 1 e 3 e a banda 18 pelas bandas 17 e 19 . Em relação às seis bandas TM/Landsat, apenas a banda 2 foi retirada por colinearidade, o que difere dos resultados obtidos por NANNI et al. (2004), onde todas as variáveis orbitais foram necessárias. Ainda segundo os autores, a área de estudo possui grande variedade de material parental, composto por material retrabalhado (arenitodiabásio, arenito-folhelho e diabásio-folhelho), sendo mais heterogenia em superfície, o que difere da área em estudo neste trabalho, mais homogenia em superfície, o que deve ser levado em consideração quanto ao menor número de variáveis selecionadas para o modelo. Exemplos de equações lineares discriminantes geradas para alguns solos com os dados de laboratório e para os dados orbitais podem ser observados na tabela 3. As equações têm por objetivo nortear e otimizar os resultados de classificação de solos utilizando dados de reflectância. Dessa forma, pressupondo o conhecimento das reflectâncias, de laboratório ou orbitais, de uma determinada amostra, sem que se saiba a qual classe de solo pertença, podem ser calculadas as bandas e alturas dos dados de laboratório ou bandas TM devidamente processadas, e aplicá-las em todas as equações. Assim, a equação discriminante do solo, cujo valor final for o maior valor resultante, terá maior probabilidade de representar a mesma classe da amostra desconhecida, otimizando assim as prospeç̧ões, auxiliando em uma identificação preliminar mais rápida, conforme Coleman e Montgomery (1990), Demattê e Garcia (1999a) e NANNi et al.(2004).

\section{Análise das equações discriminantes geradas}

Tanto as equações discriminantes de laboratório como dos dados orbitais foram geradas com $80 \%$ das amostras da área e testadas com $20 \%$ das amostras restantes, a escolha das amostras foi aleatória, determinada pelo SAS, tendo sido o procedimento testado cinquenta vezes. Após os testes, foram geradas matrizes de confusão que estão resumidas nas tabelas 4 e 5 . 
Tabela 1. Relação das variáveis preditoras utilizadas para análise discriminante das classes de solos na área de estudo

\begin{tabular}{|c|c|c|c|c|c|c|c|}
\hline \multirow{2}{*}{$\begin{array}{l}\operatorname{Bdas}\left({ }^{1}\right) \\
\mathrm{A}^{5}\end{array}$} & \multicolumn{2}{|c|}{$\lambda\left({ }^{2}\right)$} & \multicolumn{2}{|c|}{ Alturas $\left({ }^{3}\right)$} & \multirow{2}{*}{$\begin{array}{c}\lambda \\
\mathrm{nm}\end{array}$} & \multirow{2}{*}{$\begin{array}{c}\mathrm{TM}\left({ }^{4}\right) \\
\mathrm{A}\end{array}$} & \multirow{2}{*}{$\begin{array}{c}\lambda \\
\mathrm{nm}\end{array}$} \\
\hline & $B^{6}$ & $\mathrm{~nm}$ & A & $\mathrm{B}$ & & & \\
\hline B_1 $1^{7}$ & B_1 & $401-481$ & $\mathrm{H} 1^{8}$ & H1 & $469-532$ & 1 & $450-520$ \\
\hline B_3 & B_3 & $481-596$ & $\mathrm{H} 2$ & $\mathrm{H} 2$ & $532-768$ & 3 & $630-690$ \\
\hline B_4 & B_4 & $596-710$ & H3 & H3 & $768-876$ & 4 & $760-900$ \\
\hline B_5 & B_5 & $710-814$ & - & $\mathrm{H} 4$ & $876-1353$ & 5 & $1150-1750$ \\
\hline B_6 & & $814-975$ & H5 & H5 & $1353-1411$ & 7 & $2080-2350$ \\
\hline \multirow[t]{2}{*}{ B_7 } & B_7 & $975-1350$ & H6 & H6 & 1411-1439 & - & - \\
\hline & B_8 & $1350-1417$ & H7 & $\mathrm{H} 7$ & $1439-1783$ & - & - \\
\hline B_9 & & 1417 & H8 & H8 & $1860-1923$ & - & - \\
\hline B_10 & B_10 & 1471-1449 & H9 & H9 & $1923-2120$ & - & - \\
\hline B_11 & B_11 & 1449-1793 & H10 & H10 & $2120-2206$ & - & - \\
\hline B_12 & B_12 & 1793-1831 & - & H11 & $2206-2258$ & - & - \\
\hline \multirow[t]{2}{*}{ B_13 } & B_13 & 1865-1927 & H12 & H12 & $2258-2389$ & - & - \\
\hline & B_14 & 1927 & - & H13 & $2389-2498$ & - & - \\
\hline B_15 & B_15 & 1927-2102 & - & - & - & - & - \\
\hline B_17 & B_17 & 2139-2206 & - & - & - & - & - \\
\hline \multirow[t]{2}{*}{ B_19 } & B_19 & $2206-2258$ & - & - & - & - & - \\
\hline & B_20 & 2258 & - & - & - & - & - \\
\hline B_21 & B_21 & $2258-2389$ & - & - & - & - & - \\
\hline B_22 & B_22 & 2389-2498 & - & - & - & - & - \\
\hline
\end{tabular}

( $\left.{ }^{1}\right)$ Bandas selecionadas entre as 22 bandas estabelecidas, variáveis preditoras, dos modelos discriminantes dos solos da área de estudo.

$\left({ }^{2}\right)$ Comprimento de onda das bandas selecionadas para o modelo

( $\left.{ }^{3}\right)$ Alturas selecionadas entre as 13 alturas estabelecidas, variáveis preditoras, dos modelos discriminantes dos solos da área de estudo.

$\left({ }^{4}\right)$ Dados orbitais do TM-Landsat-5, selecionados entre as 6 Bandas, variáveis preditoras, dos modelos discriminantes dos solos da área de estudo.

$\left({ }^{5}\right)$ Amostras da superfície denominadas A $(0-20 \mathrm{~cm}$ de profundidade).

(6) Amostras da subsuperfície denominadas B $(80-100 \mathrm{~cm}$ de profundidade).

$\left({ }^{7}\right)$ Banda selecionada, considerada a média dos valores de reflectância entre o comprimento de onda de 401-481nm.

$\left({ }^{8}\right)$ Altura selecionada, considerada como a diferença entre o ponto de maior reflectância e o menor dentro do comprimento de onda selecionado, sendo esse valor sempre positivo.

Para os dados de laboratório das 473 amostras, 385 foram classificadas corretamente obtendo-se um acerto total de $81,4 \%$, o que perfaz uma confusão de $18,6 \%$ com 88 amostras (Tabela 4). Esses valores estão próximos aos obtidos por NANNi et al. (2004), que obteve acerto global acima dos 80\%. Demattê e GARCia (1999b) registraram acerto de $100 \%$ para dois solos estudados. Demattê et al. (2000), com dados de radiometria de laboratório, obtiveram $100 \%$ de acerto em seis unidades de solos da região de Lençóis Paulista. Em algumas classes de solos com números de observações iguais ou menores que sete, os acertos são de $100 \%$, como CXef1, LVA3, PA1, PA2, PVA1, PVA2, PVA3 e PVA4; o LVA2 com seis amostras, o acerto foi de $83,3 \%$; o PV4 com duas amostras, foi de apenas $50 \%$ de acerto (Tabela 4). Esse fato está de acordo com Oliveira et al. (1982), os quais consideram que sete deve ser o número mínimo de amostras por classe de solo, para que as análises estatísticas não tenham influência marcante no erro padrão da média.
NANNi (2000) considera também que as classes com número reduzido de amostras podem não contemplar a variabilidade existente na classe, ou possuir características muito semelhantes a outra classe. BEN-Dor (2002) descreveu a importância de ter amostras representativas de toda uma área na qual se pretende elaborar modelos. O PVA1 é a única classe com $100 \%$ de acerto e o número mínimo de observações igual a sete (Tabela 4). Para o PV4, obteve-se um acerto e um erro de $50 \%$. Nesse solo foram feitas apenas duas observações na área de estudo, sendo confundido no teste das equações com o LV4, que difere apenas na textura na superfície (54 g kg-1 de argila) maior que a do PV4 $\left(34,5 \mathrm{~g} \mathrm{~kg}^{-1}\right.$ de argila), sendo a relação textural um dos fatores mais importantes na diferenciação entre as duas ordens, Latossolos e Argissolos (Tabelas 2 e 5). O pequeno número de observações dentro de uma mesma classe pode elevar muito o erro, como verificado por NANNI (2000). 


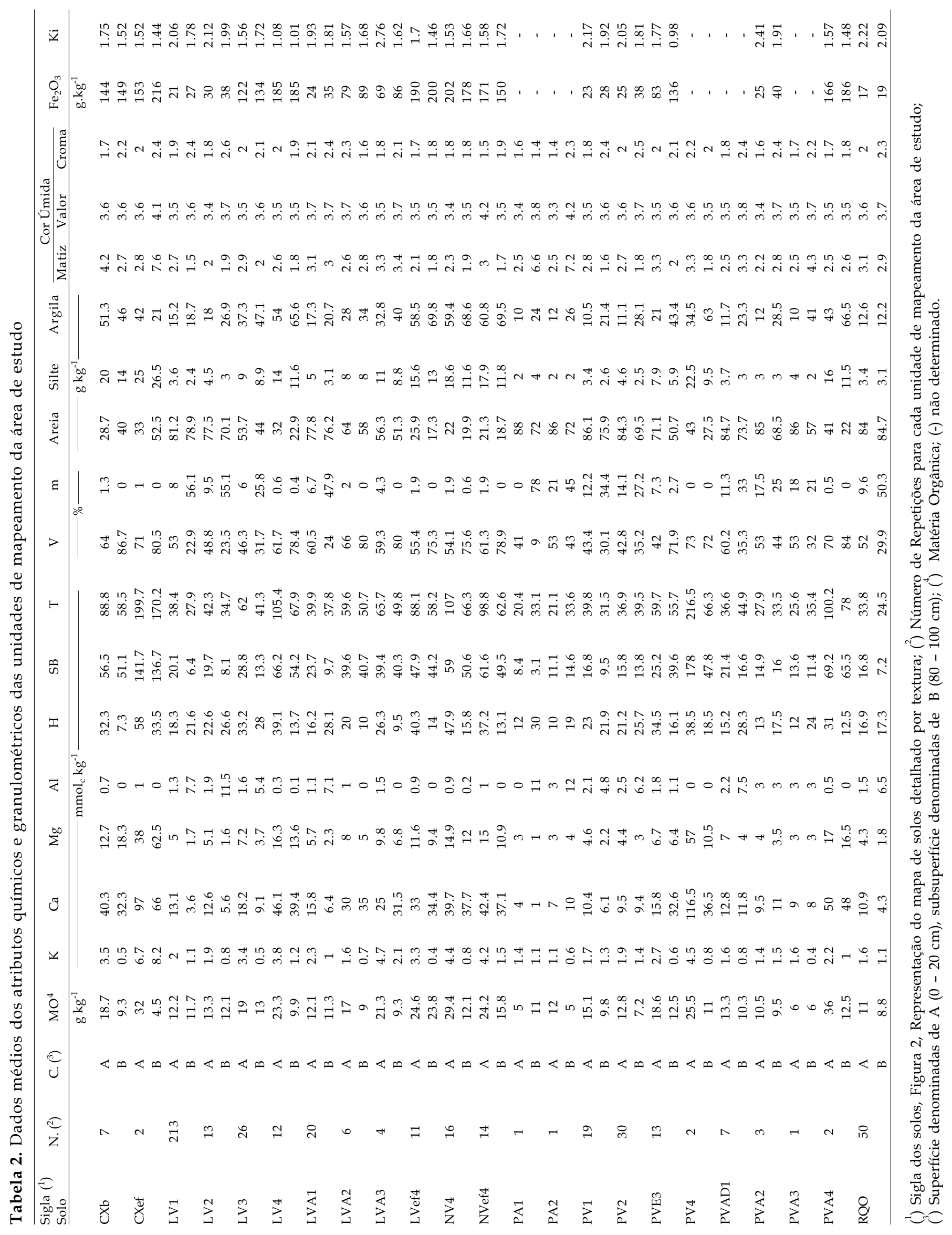




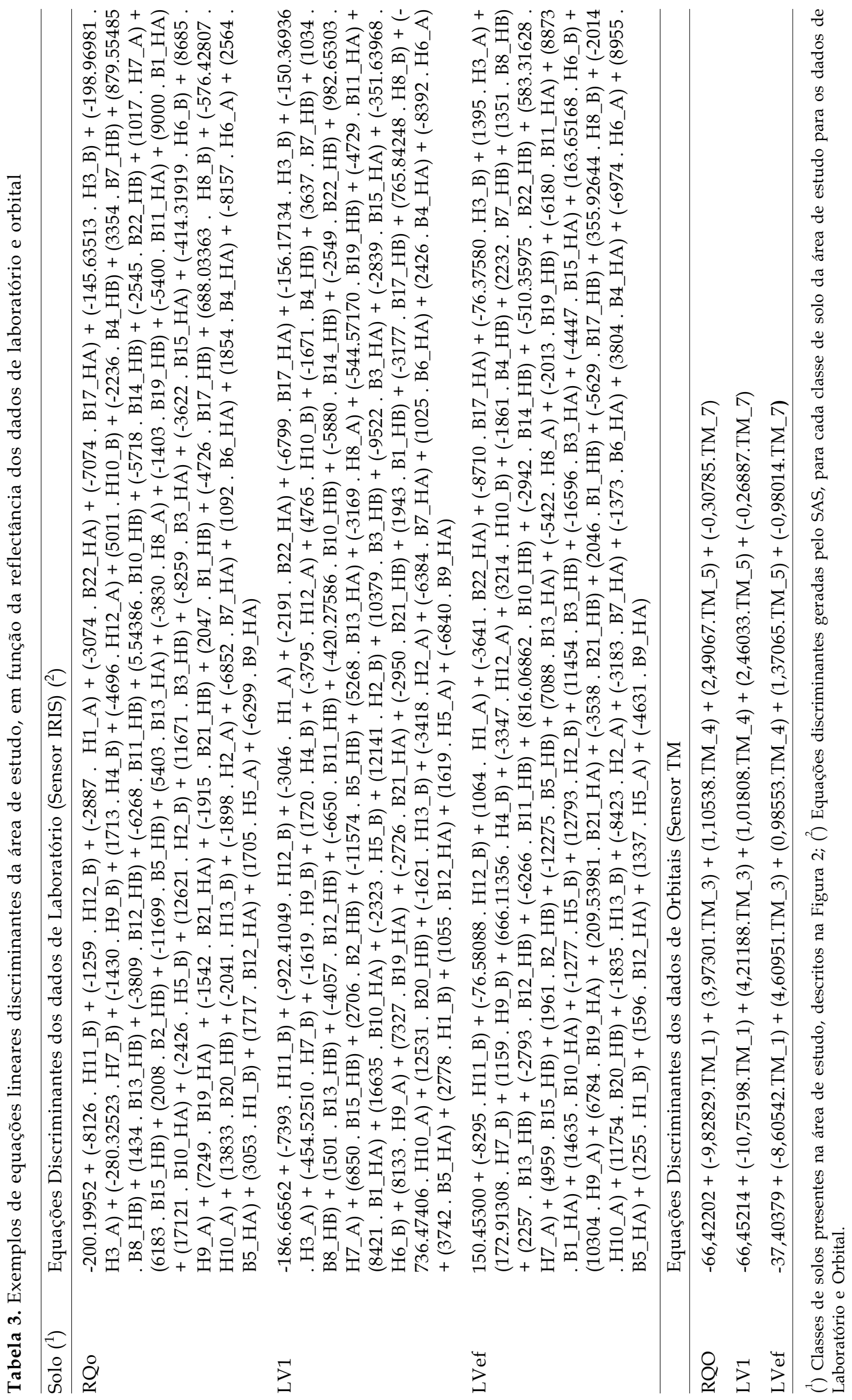


Tabela 4. Resultados da análise discriminante, número de observações, classificação e erro para os dados obtidos das bandas e alturas de 473 amostras da área de estudo

\begin{tabular}{|c|c|c|c|c|c|c|}
\hline \multirow{2}{*}{ Solo $\left({ }^{1}\right)$} & \multirow{2}{*}{$\begin{array}{c}\text { Total de } \\
\text { Amostras }\left({ }^{2}\right)\end{array}$} & \multicolumn{2}{|c|}{ Acerto $\left({ }^{3}\right)$} & \multicolumn{2}{|c|}{ Erro $\left({ }^{4}\right)$} & \multirow{3}{*}{ Solos confundidos $\left({ }^{5}\right)$} \\
\hline & & Amostras & \multicolumn{3}{|c|}{ Amostras } & \\
\hline & & & $\%$ & & $\%$ & \\
\hline $\mathrm{CXb}$ & 7 & 5 & 71,4 & 2 & 28,57 & LV3(1), NV(1) \\
\hline CXef1 & 2 & 2 & 100,0 & - & - & - \\
\hline LV1 & 213 & 182 & 85,4 & 31 & 14,55 & LV2(3), LVA1(10), PV1(10), PV2(3), RQo(5) \\
\hline LV2 & 13 & 9 & 69,2 & 4 & 30,77 & LV1(1), LVA1(1), PV1(1), PV2(1) \\
\hline LV3 & 26 & 21 & 80,7 & 5 & 19,23 & LV4(2), LVef(2), NVef(1) \\
\hline LV4 & 12 & 12 & 100,0 & - & - & - \\
\hline LVA1 & 20 & 16 & 80,0 & 4 & 20,00 & LV1(1), PVA1(1), RQo(2) \\
\hline LVA2 & 6 & 5 & 83,3 & 1 & 16,67 & LV3(1) \\
\hline LVA3 & 4 & 4 & 100,0 & - & - & - \\
\hline LVef & 11 & 8 & 72,7 & 3 & 27,27 & $\operatorname{LV} 3(2), \operatorname{NVef}(1)$ \\
\hline NV & 16 & 10 & 62,5 & 6 & 37,50 & LV3(1), LV4(2), NVef(2), PVA4(1) \\
\hline NVef & 14 & 12 & 85,7 & 2 & 14,29 & $\operatorname{LVef}(2)$ \\
\hline PA1 & 1 & 1 & 100,0 & - & - & - \\
\hline PA2 & 1 & 1 & 100,0 & - & - & - \\
\hline PV1 & 19 & 15 & 78,9 & 4 & 21,05 & LV1(2), PV2(1), RQo(1) \\
\hline PV2 & 30 & 25 & 83,3 & 5 & 16,67 & LV1(2), LV3(1), PV3(1), PVA1(1) \\
\hline PV3 & 13 & 12 & 92,3 & 1 & 7,69 & LV3(1) \\
\hline PV4 & 2 & 1 & 50,0 & 1 & 50,00 & LV4(1) \\
\hline PVA1 & 7 & 7 & 100,0 & - & - & - \\
\hline PVA2 & 3 & 3 & 100,0 & - & - & - \\
\hline PVA3 & 1 & 1 & 100,0 & - & - & - \\
\hline PVA4 & 2 & 2 & 100,0 & - & - & - \\
\hline RQo & 50 & 31 & 62,0 & 19 & 38,00 & LV1(12), LVA1(1), PV1(3), PVA1(2), PVA3(1) \\
\hline Total & 473 & 385 & 81,4 & 88 & 18,6 & - \\
\hline
\end{tabular}

(1) Classes de solos presentes na área de estudo, descritos na figura 2, deste trabalho.

$\left({ }^{2}\right)$ Total de observações de cada classe.

$\left({ }^{3}\right)$ Número de amostras e porcentagem classificadas corretamente pela equação discriminante para cada classe de solo.

$\left({ }^{4}\right)$ Número de amostras e porcentagem classificadas erroneamente pela equação discriminante para cada classe de solo.

$\left({ }^{5}\right)$ Classes de solos que foram confundidas no processo de classificação pelas equações discriminantes.

Para se observar se no acerto global haveria alguma influência devido aos solos com numero de mostras inferior a sete, os mesmos foram retirados. Das 451 amostras restantes, as equações discriminantes classificaram corretamente 385 amostras com um acerto global de $80,9 \%$ e uma confusão de $19,3 \%$, podemos considerar que essas amostras não estão influenciando os resultados. Vários solos tiveram acertos próximos ou maiores que 80\%, LV1, LV3, LVA1, LVA2, NVef, PV1, PV2, PV3 (Tabela 4). Das quatorze observações do NVef, apenas duas foram confundidas com o LVef, sendo esses solos muito semelhantes (Tabela 2), diferindo no campo pela descrição morfológica e principalmente presença de serosidade no NVef (EMBRAPA, 1999). O LV1 é o solo com maior representatividade na área de estudo, com 213 amostras, sendo 182 corretamente classificadas e 31 confundidas (Figura 2 e Tabela 4). Das 31 amostras confundidas, houve dois solos com maior frequência, o LVA1 com 10 amostras, que difere do LV1 apenas na cor em subsuperfície, com matiz 3.0 YR (LVA1) e 1.5 YR (LV1) (Tabela 2), e o PV1, também com 10 amostras, difere do LV1 na diferença textural das superfícies no Argissolo (Tabela 2). 
Tabela 5. Resultados da análise discriminante, número de observações, classificação e erro para os dados obtidos das 6 bandas do TM-Landsat 5 nas 473 amostras da área de estudo

\begin{tabular}{|c|c|c|c|c|c|c|}
\hline \multirow{2}{*}{ Solo $\left({ }^{1}\right)$} & \multirow{2}{*}{$\begin{array}{c}\text { Total de } \\
\text { Amostras }\left({ }^{2}\right)\end{array}$} & \multicolumn{2}{|c|}{ Acerto $\left({ }^{3}\right)$} & \multicolumn{2}{|c|}{ Erro $\left({ }^{4}\right)$} & \multirow[t]{2}{*}{ Solos confundidos $\left({ }^{5}\right)$} \\
\hline & & Amostras & $\%$ & Amostras & $\%$ & \\
\hline $\mathrm{CXb}$ & 7 & - & - & 7 & 100,0 & $\begin{array}{l}\text { LVA3(1), LVef(2), NV(1), NVef(1), PV4(1), } \\
\text { PVA4(1) }\end{array}$ \\
\hline CXef1 & 2 & 2 & 100,0 & - & - & - \\
\hline LV1 & 213 & 148 & 69,4 & 65 & 30,5 & $\begin{array}{l}\text { LV2(7), LVA1(7), LVA2(17), PA1(3), PV1(2), } \\
\text { PV2(1), PV3(2), PV4(1), PVA1(5), PVA2(9), } \\
\text { PVA3(4), RQo(7) }\end{array}$ \\
\hline LV2 & 13 & 1 & 7,7 & 12 & 92,3 & $\begin{array}{l}\text { LV1(2), LVA1(1), LVef(1), PA2(1), PV1(1), } \\
\text { PV2(1), PV4(2), PVA2(2), PVA3(1) }\end{array}$ \\
\hline LV3 & 26 & 4 & 15,4 & 22 & 84,6 & $\begin{array}{l}\text { CXB(1), LV4(5), LVA3(4), LVef(2), NV(1), } \\
\text { NVef(1), PA2(1),PV2(1), PV3(1), PV4(2), } \\
\text { PVA1(1), PVA4(2) }\end{array}$ \\
\hline LV4 & 12 & 1 & 8,3 & 11 & 91,6 & $\begin{array}{l}\text { CXef1(2), LV3(2), LVef(2), NV(1), PA2(1), } \\
\text { PV4(1), PVA4(2) }\end{array}$ \\
\hline LVA1 & 20 & 1 & 5,0 & 19 & 95,0 & $\begin{array}{l}\text { LV1(5), LVA2(3), PA1(1), PA2(1), PV4(1), } \\
\text { PVA2(3), PVA3(4), RQo(1) }\end{array}$ \\
\hline LVA2 & 6 & 2 & 33,3 & 4 & 66,6 & LV1(1), PA2(1), PVA1(1), PVA3(1) \\
\hline LVA3 & 4 & 3 & 75,0 & 1 & 25,0 & $\operatorname{LVef}(1)$ \\
\hline LVef & 11 & 3 & 27,3 & 8 & 72,7 & $\begin{array}{l}\text { CXef1(1), LV4(2), LVA3(1), NV(1), NVef(1), } \\
\text { PV4(2) }\end{array}$ \\
\hline NV & 16 & 2 & 12,5 & 14 & 87,5 & $\begin{array}{l}\text { CXef1(1), LV4(3), LVef(4), NVef(4), PV4(1), } \\
\text { PVA1(1) }\end{array}$ \\
\hline NVef & 14 & 8 & 57,1 & 6 & 42,8 & CXB(1), LV4(1), LVA3(1), NV(1), PV4(2) \\
\hline PA1 & 1 & 1 & 100,0 & & - & - \\
\hline PA2 & 1 & 1 & 100,0 & & - & - \\
\hline PV1 & 19 & 1 & 5,3 & 18 & 94,7 & $\begin{array}{l}\text { LV1(5), LV2(3), LVA1(1), LVA2(2), PV2(2), } \\
\text { PVA2(2) PVA3(2), RQo(1) }\end{array}$ \\
\hline PV2 & 30 & 2 & 6,7 & 28 & 93,3 & $\begin{array}{l}\text { LV1(1), LV2(3), LV3(1), LVA1(1), LVA2(3), } \\
\text { LVA3(1), PV1(1), PV3(3), PVA1(1), PVA2(5), } \\
\text { PVA3(8) }\end{array}$ \\
\hline PV3 & 13 & 1 & 7,7 & 12 & 92,3 & $\begin{array}{l}\text { CXef1(1), LV1(1), LV3(2), LV4(1), LVA3(2), } \\
\text { LVef(2), PA2(1), PV2(1), PVA4(1) }\end{array}$ \\
\hline PV4 & 2 & & - & 2 & 100,0 & LV3(1), NVef(1) \\
\hline PVA1 & 7 & 2 & 28,6 & 5 & 71,4 & LVA2(1), PA1(2), PVA2(1), PVA3(1) \\
\hline PVA2 & 3 & 1 & 33,3 & 2 & 66,7 & LVA1(1), PVA3(1) \\
\hline PVA3 & 1 & 1 & 100,0 & - & - & - \\
\hline PVA4 & 2 & 1 & 50,0 & 1 & 50,0 & LV4(1) \\
\hline RQo & 50 & 5 & 10,0 & 45 & 90,0 & $\begin{array}{l}\text { CXef1(2), LV1(21), LV2(2), LVA1(2), } \\
\text { LVA2(3), PA1(2), PV1(1), PV2(2), PVA1(4), } \\
\text { PVA2(2), PVA3(3), PVA4(1) }\end{array}$ \\
\hline Total & 473 & 191 & 40,4 & 282 & 59,6 & - \\
\hline
\end{tabular}

$\left({ }^{1}\right)$ Classes de solos presentes na área de estudo, descritos na Figura 2, deste trabalho.

( $\left.{ }^{2}\right)$ Total de observações de cada classe.

( $\left.{ }^{3}\right)$ Número de amostras e porcentagem classificadas corretamente pela equação discriminante para cada classe de solo.

$\left({ }^{4}\right)$ Número de amostras e porcentagem classificadas erroneamente pela equação discriminante para cada classe de solo.

$\left({ }^{5}\right)$ Classes de solos que foram confundidas no processo de classificação pelas equações discriminantes. 
A confusão entre o LV1 e o RQo, com cinco observações, é justificável, uma vez que as texturas estão muito próximas de $15 \mathrm{~g} \mathrm{~kg}^{-1}$ de argila, que define a mudança de textura arenosa para média (Tabela 2). $\mathrm{O}$ PV2 e o LV2 também se confundem, embora com menor frequência, com o LV1, visto que a variabilidade dos atributos químicos e físicos dentro de uma mesma classe pode estar próxima aos limites que diferenciam os solos encontrados na área de estudo, diferindo principalmente nas texturas (Tabelas 2 e 5), o que está de acordo com NANNI et al. (2004). As mesmas considerações se aplicam para os PV1, PV2, PV3, LV3, LVA2 e LVA1, confundidos com solos de texturas muito semelhantes independentemente da ordem (Tabela 4 e 2). A menor porcentagem de acerto foi de $62 \%$, com 31 amostras classificadas corretamente das 50 observações do RQo (Tabela 4). Esse solo foi confundido em 19 observações com solos de textura muito semelhante à arenosa ou média, provenientes do arenito com cimento argiloso, justificando a confusão com os demais solos, com exceção de uma amostra de PVA3 com textura arenosa na superfície e argilosa na subsuperfície. Outros solos tiveram porcentagens de acertos entre $62,5 \%$ e $73 \%$; em ordem crescente de acertos, há os solos NV, LV2, LVef e $\mathrm{CXb}$ e também foram confundidos com outros solos com características muito semelhantes principalmente quanto às características físicas e químicas para o NV, LVef e CXb para ambas as superfícies de amostragem e no caso do LV2 com solos com texturas médias (Tabelas 4 e 2). Para os dados orbitais das 473 amostras, 191 foram classificadas corretamente, obtendo-se acerto total de $40,4 \%$, o que perfaz um erro de $59,6 \%$ com 282 amostras (Tabela 5). Esses valores estão próximos aos obtidos por NANNI (2000) com acerto global próximo de $40 \%$.

Demattê et al. (2000) observaram acerto de 99, $3 \%$ para 26 unidades de mapeamento da região de Piracicaba, avaliada por imagens Landsat. Para as classes com número de observações menores ou iguais a sete, há os CXef, PA1, PA2 e PVA3, com $100 \%$ de acerto. Verificou-se também $100 \%$ de confusão paras as classes CXb e PV4, apesar de serem confundidos com solos de texturas semelhantes, mais argilosas (Tabelas 5 e 2). O PVA4, para as suas duas observações, com acerto de $50 \%$, foi confundido com o LV4; nesses solos, para a superfície não há diferenças (Tabela 2). Os demais solos com menor número de observações, LVA2, LVA3, PVA2 e PVA1, obtiveram baixas porcentagens de acerto, e na maioria dos casos foram confundidos com solos semelhantes pela textura, para superfície, (Tabelas 5 e 2). Os resultados mais satisfatórios para o nível orbital são para as classes LV1 com 69,4\% de acerto e para NVef com $57,1 \%$. O LV1 tem como material de origem o arenito com cimento argiloso e textura média ( $15 \mathrm{a} 25 \mathrm{~g} \mathrm{~kg}^{-1} \mathrm{de}$ argila), predominando em áreas de topo do relevo. Com exceção do PV3, nos demais solos confundidos no teste da equação discriminante dos dados orbitais, apresentam características físicas em superfície foram semelhantes (Tabela 5 e 2). Tal fato se repete para o solo NVef, o qual é originado do basalto, com em relevos mais ondulados e textura mais argilosa. A discriminação de solos desenvolvidos de basalto está de acordo com Nanni e Demattê (2001a), que estudaram solos do Paraná. É importante destacar que, apesar dos dados orbitais, não observou maior porcentagem de acerto e os resultados podem ser considerados satisfatórios, uma vez que a maioria dos solos confundidos, em superfície, é semelhante em suas classes texturais, podendo ser agrupados em dois grandes grupos: o primeiro de textura arenosa - média, provenientes do arenito de cimento argiloso; o segundo mais argiloso originado do basalto. NANNI (2000) verificou que classes de solos com teores de ferro mais elevados, material de origem diabásio, tiveram pouca ou nenhuma confusão com as classes oriundas do arenito. Mesmo em condições de campo, para a porção superficial, as características químicas e físicas são muito semelhantes dentro de cada um desses dois grandes grupos, tais como teores de ferro, matéria orgânica, argila, areia e cor, o que dificultaria sua separação, concordando com NANNI (2000). A utilização das equações discriminantes dos dados orbitais é uma ferramenta importante em uma primeira avaliação mais rápida de solos de diferentes regiões do país, concordando com Coleman e MONTGOMERY (1990).

\section{CONCLUSÕES}

1. Com metodologia adequada, é possível discriminar solos por meio do sensoriamento remoto em laboratório, utilizando-se amostras de terra de superfície e subsuperficie.

2. Para os dados de laboratório das áreas com solos mais homogêneos, o número de variáveis preditoras tende a ser maior que para áreas com solos mais heterogêneos, devido à maior dificuldade de diferenciá-los.

3. Das setenta variáveis iniciais, apenas cinquenta e seis foram selecionadas para o modelo, com maior participação das variáveis em subsuperfície sendo esses dados de maior importância na classificação das unidades de solos.

4. Para os dados de laboratório, as equações obtiveram um acerto de $81 \%$, contra $40 \%$ dos dados das equações orbitais.

5- Os sensores orbitais auxiliam na discriminação das características de superfície dos solos, podendo ser utilizados na diferenciação de padrões de texturas. 


\section{AGRADECIMENTOS}

À Fundação de Amparo à Pesquisa do Estado de São Paulo, pela aquisição do espectroradiômetro IRIS, (proc. n 95/6259-6), pela bolsa de Doutorado do primeiro autor (proc. n ${ }^{\circ} 99 /$ 04325-2); agradecimentos também ao CNPq pela bolsa de pesquisador do segundo autor (proc. n. ${ }^{\circ}$ 300371/96-9).

\section{REFERÊNCIAS}

AFIFI, A.; CLARK, V.A.; MAY,S. Computer-aided multivariate analysis. Boca Raton: Chapman \& Hall, 2004. 419p.

BAPTISTA, G.M.M.; MADEIRA NETO, J.S.; CARVALHO JUNIOR, O.A.; MARTINS, E.S. Mapeamento dos teores de argila de solos tropicais, por meio de dados de sensoriamento remoto hiperespctral (compact disc). In: CONGRESSO BRASILEIRO DE CIÊNCIA DOSOLO, 27., 1999 Brasília. Anais... Planaltina: Embrapa Cerrados, 1999.

BAUMGARDNER, M.F.; SILVA, L.F.; BIEHL, L.L.; STONER, E.R. Reflectance properties of soils. Advances in Agronomy, v.38, p.1-44, 1985.

BEN-DOR, E. Quantitative remote sensing of soil properties. Advances in Agronomy, v.75, p.173-243, 2002.

CAMARGO, M.N.; KLANT, E.; KAUFFMAN, J.H. Classificação de solos usada em levantamentos pedológicos no Brasil. Campinas. Boletim Informativo da Sociedade Brasileira de Ciência do Solo, v.12, p.11-13, jan./abr., 1987.

COLEMAN, T.L.; AGBU, P.A.; MONTGOMERY, O.L. Spectral differentiantion of soils and soil properties: is it possible from space plataforms? Soil Science, v.155, p.283-293, 1993.

COLEMAN, T.L.; MONTGOMERY,O.L. Assessment of spectral characteristics for differentiating among soil categories in the southeastern united States. Photogrammetric Engineering \& Remote Sensing, v.52, p.1659-1663, 1990.

DEMATTÊ, J.A.M. O pedólogo e agricultura de precisão. Boletim Informativo da Sociedade Brasileira de Ciência do Solo, Viçosa, v.26, n.1, Jan/Mar, p.17-19, 2001.

DEMATTÊ, J.A.M., HUETE A.R., FERREIRA Jr. L.G., ALVES M.C., NANNI M.R., CERRI C.E. Evaluation of tropical soils through ground and orbital sensors. In: INTERNATIONAL CONFERENCE GEOSPATIAL INFORMATION IN AGRICULTURE AND FORESTRY, 2., 2000. Proceeding...Lake Buena Vista: Erim, 2000. v.2, p. 34-41,

DEMATTÊ, J.A.M.; GARCIA, G.J. Alteration of soil properties through a weathering sequence as evaluated by spectral reflectance. Soil Science Society of America Journal, v.63, p. 327-342, 1999a.

DEMATTÊ, J.A.M.; GARCIA, G.J. Avaliação de atributos de Latossolo Bruno e de Terra Bruna Estruturada da região de Guarapuava, Paraná, por meio de sua energia refletida Revista
Brasileira de Ciência do Solo, v.23, p.343-355, 1999b.

DEMATTÊ, J.A.M.; NANNI, M.R. Weathering sequence of soils developed from basalt as evaluated by laboratory (IRIS), airborne (AVIRIS) and orbital (TM) sensors. International Journal of Remote Sensing, v.24, p.4715- 4738, 2003.

EMPRESA BRASILEIRA DE PESQUISA AGROPECUÁRIA. Centro Nacional de Pesquisa de Solos. Sistema Brasileiro de Classificação de Solos. Brasília: Serviço de Produção, 1999. $412 \mathrm{p}$.

EPIPHANIO, J.C.N.; FORMAGGIO, A.R. Abordagens de uso de número digital e de reflectância em sensoriamento remoto com dados de satélites. In: SIMPÓSIO BRASILEIRO DE SENSORIAMENTO REMOTO, 5., Natal, 1988. Anais... São José dos Campos: INPE, 1988. p.400-405.

EPIPHANIO, J. C. N.; FORMAGGIO, A. R.; VALERIANO, M.; OLIVEIRA, J. B. Comportamento espectral de solos do Estado de São Paulo. São José dos Campos: INPE, 1992. 131p.

FIORIO, P.R.; DEMATTÊ, J.A.M.; SPAROVEK, G. Cronologia do uso da terra e seu impacto ambiental na microbacia hidrográfica do Córrego do Ceveiro, Piracicaba (SP). Pesquisa Agropecuária Brasileira, v.35, n.4, p.671-679, abr. 2000.

FIORIO, P.R.; DEMATTÊ, J.A.M. Orbital and laboratory spectral data optimize soil analysis. Scientia Agricola, v.66, n.2, p.250257, Mar/Abr. 2009.

FORMAGGIO, A.R.; EPIPHANIO, J.C.N.; VALERIANO, M.M.; OLIVEIRA, J.B. Comportamento espectral $(450-2.450 \mathrm{~nm})$ de solos tropicais de São Paulo. Revista Brasileira de Ciência do Solo, v.20, p.467-474, 1996.

GEOPHYSICAL ENVIRONMENTAL RESEARCH CORP, GER. Mark V Dual Field of View IRIS Manual. Version 1.3. New York: Milbook, 1996. 63p.

INSTITUTO DE PESQUISAS TECNOLÓGICAS. IPT. Divisão de Minas e Geologia Aplicada. Mapa geológico do Estado de São Paulo. São Paulo, 1981. Escala 1:1000.000.

INSTITUTO NACIONAL DE PESQUISAS ESPACIAIS. INPE. Tutorial Spring: spring básico. São José dos Campos: INPE, 1999. não paginado.

KRAVCHENKO, A.N.; BOLLERO, G.A.; OMONODE, R.A.; BULLOCK, D.G. Quantitative mapping of soil drainage classes using topographical data and soil electrical conductivity. Soil Science Society of America Journal, v.66, p.235-243, 2002.

LABSPHERE, REFLECTANCE CALIBRATION LABORATORY. Spectral reflectance target calibrated from $\mathbf{0 . 2 5 - 2 . 5} \mathrm{mm}$ reported in $0.050 \mathrm{~mm}$ intervals. Sutton, 1996. 5p.

LEMOS, R.C.; SANTOS, R.D. Manual de descrição e coleta de solo no campo. 3.ed. Campinas: Sociedade Brasileira de Ciência do Solo, 1996. 84p.

NANNI, M.R.; DEMATTE, J.A.M.; FIORIO, P. R. Análise discriminante dos solos por meio da resposta espectral no nível terrestre. Pesquisa Agropecuária Brasileira, v.39, p.995-1006, 2004. 
NANNI, M.R.; DEMATTÊ, J.A.M. Is it possible estimate physicalchemical soil attributes by using laboratory and orbital sensors. In: INTERNATIONAL CONFERENCE OF GEOSPATIAL INFORMATION IN AGRICULTURE AND FORESTRY, 3., 2001, Denver, Colorado. Proceedings... 2001b (compact disc).

NANNI, M.R.; DEMATTÊ, J.A.M. Quantification and discrimination of soils developed from basalt as evaluated by terrestrial, airborne and orbital sensors (compact disc). In:SIMPÓSIO BRASILEIRO DE SENSORIAMENTO REMOTO, 10., 2001, Foz do Iguaçu. Anais... São José dos Campos: INPE, 2001a. p.1441-1449.

NANNI, M.R. Dados radiométricos obtidos em laboratório e no nível orbital na caracterização e mapeamento de solos. 2000, 365p. Tese (Doutorado)- ESALQ/USP, Piracicaba.

NICODEMUS, F.E.; RICHMOND, J.C.; HSIA, J.J.; GINSBERG, I.W.; LIMPERIS, T. Geometrical considerations and nomenclature for reflectance. Washington, D.C.: US Department of Commerce, 1977. 52 p. (NBS Monograph, 160)

ODEH, I.O.A.; MAcBRATNEY, A.B. Using AVHRR images for spatial prediction of clay content in the lower Namoi Valley of eastern Australia. Geoderma, p.237-254, 2000.

OLIVEIRA, J.B.; MENCK, J.R.F.; BARBIERI, J.L. et al. Levantamento pedológico semidetalhado do Estado de São Paulo : quadrícula de Araras. Campinas : IAC, 1982. 180p. (Boletim Técnico, 71)

RAIJ, B. van; QUAGGIO, J.A. Métodos de análise de solo para fins de fertilidade. Campinas: IAC, 1983. 40p. (Boletim técnico, 81)

SENTELHAS, P.C.; MARIN, F.R.; PEREIRA, R.; ANGELOCCI, L.R.; VILA NOVA, N.A.; BARBIERI, V. Análise de dados climáticos e do balanço hídrico climatológico de Piracicaba (1917-1997). Piracicaba: DFM/ESLQ/USP, 1998, 81p.

SINOWSKI, W.; AUERSWALD, K. Using relief parameters in a discriminant analysis to stratify geological areas with different spatial variability of soil properties. Geoderma, v.89, p.113-128, 1999.

STATISTICAL ANALYSISSYSTEM INSTITUTE. SAS, software: user's guide, version 8.2, Cary, 1999. 291p.

STONER, E.R.; BAUMGARDNER, M.F. Characteristics variations in reflectance of surface soils. Soil Science Society of America Journal, v.45, p.1161-1165, 1981.

TANRÉ, D.; HOLBEN, B.N.; KAUFMAN, Y.J. Atmospherie correction algorithm for NOAA-AVHRR products: theory and application. IEEE Transactions Geoscience Remote Sensing, v.30, p.231-248. 1992.

WEBSTER, R.; BURROUGH, P.A. Multiple discriminant analysis in soil survey. Journall of Soil Science, v.23, p.120134, 1974.

WEBSTER, R.; OLIVER, M.A. Statistical methods in soil and land resource survey. Oxford: Oxford University Press, 1990. 316p.

ZULLOJr, J. Correção atmosférica de imagens de satélite e aplicações. 1994. 191p. Tese (Doutorado)-Universidade de Campinas. 